Journal of Patient-Centered

Volume 4

Issue 4 -- Health Disparities and Inequities: Part

Article 24 I

$11-6-2017$

\title{
Robustness of a Newly Proposed Risk Schema for Lymphatic Dissemination in Endometrioid Endometrial Cancer
}

Danielle M. Greer

Jessica J.F. Kram

Callie M. Cox Bauer

Scott A. Kamelle

Follow this and additional works at: https://aah.org/jpcrr

Part of the Diagnosis Commons, Hemic and Lymphatic Diseases Commons, Oncology Commons, Surgical Procedures, Operative Commons, Urogenital System Commons, and the Women's Health Commons

\section{Recommended Citation}

Greer DM, Kram JJ, Cox Bauer CM, Kamelle SA. Robustness of a newly proposed risk schema for lymphatic dissemination in endometrioid endometrial cancer. J Patient Cent Res Rev. 2017;4:262.

Published quarterly by Midwest-based health system Advocate Aurora Health and indexed in PubMed Central, the Journal of Patient-Centered Research and Reviews (JPCRR) is an open access, peer-reviewed medical journal focused on disseminating scholarly works devoted to improving patient-centered care practices, health outcomes, and the patient experience. 
Conclusion: Postpartum hypertension is more recognized, and readmissions are becoming more common. We increased efforts to optimize medical management of hypertension and reduce preventable readmissions. Improvement in discharge instructions for patients did not decrease overall admission for postpartum hypertension but may have improved overall patient care. Overall cost analysis would be beneficial to see further economic impact.

\section{Robustness of a Newly Proposed Risk Schema for Lymphatic Dissemination in Endometrioid Endometrial Cancer}

Danielle M. Greer, Jessica J.F. Kram, Callie M. Cox Bauer, Scott A. Kamelle

Aurora University of Wisconsin Medical Group; Center for Urban Population Health; Department of Obstetrics and Gynecology, Brook Army Medical Center; Aurora Gynecologic Oncology

Background: Surgical management for endometrioid endometrial cancer (EEC) includes complete lymph node dissection for all patients at risk of lymphatic dissemination. The standard risk schema, defined by Mayo Clinic, identifies low-risk patients as those with grade $1 / 2$ EEC, myometrial invasion $(\mathrm{MI}) \leq 50 \%$, and tumor diameter (TD) $\leq 2 \mathrm{~cm}$. We recently proposed (and published) a risk schema containing modified forms of grade, MI and TD that suggests a significant decrease in false-negative rate and need for lymphadenectomy in low-risk women.

Purpose: Evaluate robustness of our proposed schema for lymphatic dissemination risk stratification in a subsequent EEC patient cohort. Methods: We retrospectively applied the proposed schema to patients diagnosed with stage I-III EEC during 2014-2015 who underwent pelvic and/or para-aortic lymph node removal. Cancer Registry data were confirmed via chart review. Consistent with the cohort studied during model development, the validation cohort included non-Hispanic white or black patients with complete data describing TD ( $\leq 50 \mathrm{~mm}$ or $>50 \mathrm{~mm})$, MI $(\leq 33 \%,>33 \%$ to $\leq 66 \%$, or $>66 \%$ ) and grade (1 or $2-3)$.

Results: In the validation cohort, 29 (11.7\%) of the 247 EEC patients were node-positive (vs $9.2 \%$ of 737 patients in the development cohort). Risk stratification using the proposed schema produced similar false-positive rates during model development (57.2\%) and validation $(54.6 \%)$, both $20 \%$ lower than when using the standard schema $(76.2 \%$ and $74.3 \%$, respectively). False-negative rates, however, were noticeably different between development and validation cohorts using both the proposed $(0 \%$ and $13.8 \%)$ and standard $(1.47 \%$ and $6.90 \%)$ schemas, suggesting a shift toward lowrisk classification in node-positive patients of the validation cohort. Conclusion: Application of the proposed risk stratification schema to an alternative patient cohort verified the utility of modified risk criteria, including TD with 50-mm cutoff, for identifying low-risk EEC patients who may not require node evaluation. However, in the validation cohort, greater prevalence of lymph node metastasis and low-risk classification of node-positive patients was observed. Discrepancy between cohorts is likely due to greater utilization of sentinel lymph node mapping during the validation period, allowing for increased detection of low-volume metastases. Continued model development and validation is needed, especially to account for the increased sensitivity of new technologies.
FMT Placed by Colonoscopy: Systematic Review and Meta-Analysis

Nadia Huq, Veena Kumaravel, Aboud Affi, Maharaj Singh

Departments of Internal Medicine and Gastroenterology, Aurora Sinai Medical Center; Aurora Research Institute

Background: Fecal transplants are successful in the treatment of recurrent or refractory Clostridium difficile infections (CDI), but there is no consensus on the best method of instillation. Studies have shown greater success with lower gastrointestinal tract placement, but technical aspects of placement are not validated.

Purpose: This review aims to identify common traits and procedural techniques of successful fecal microbiota transplant (FMT) therapy via colonoscopy.

Methods: An electronic search was conducted using OVID Medline and PubMed for articles published from January 2010 to January 2016. The primary outcome of interest was cure by FMT placed via colonoscopy.

Results: Of the 337 articles reviewed, we included 24 studies, from which 11 case reports were excluded from data analysis. The resultant data included 366 patients (64\% female). Point estimate for cure of CDI after FMT for patients over 65 years of age $(9 \%)$ was $84.6 \%$ (95\% confidence interval [CI]: $0.58-0.96 ; \mathrm{P}<0.016)$, cure over the age of 18 with no upper limit specified on age $(74 \%)$ was $85.4 \%$ (95\% CI: $0.76-0.91 ; \mathrm{P}<0.001)$ and, for those identified strictly as $18-65$ years old (17\%), cure was $93 \%$ (95\% CI: $0.83-$ $0.98 ; \mathrm{P}<0.001)$. Patients who stopped antibiotics at least 48 hours prior to FMT (37\%) had a cure rate of $86 \%$ (95\% CI: $0.78-0.91$; $\mathrm{P}<0.001)$ compared to $95 \%(95 \% \mathrm{CI}: 0.90-0.98 ; \mathrm{P}<0.001)$ in patients who stopped antibiotics at least 24 hours prior to FMT $(43 \%)$ and $81 \%(95 \%$ CI: $0.53-0.94 ; \mathrm{P}<0.035)$ in those who stopped less than 24 hours prior to FMT (15\%). In studies that specified use of GoLYTELY ${ }^{\circledR}$ prep prior to colonoscopy (58\%), cure was $91 \%$ (95\% CI: 0.85-0.95; $\mathrm{P}<0.001$ ); whereas those using a split $2-\mathrm{L}$ polyethylene glycol prep (21\%) had $79 \%$ cure $(95 \%$ CI: $0.61-0.90$; $\mathrm{P}<0.004)$. Placement of FMT throughout the colon $(6.8 \%)$ had $96 \%$ cure (95\% CI: $0.77-0.99 ; \mathrm{P}<0.002)$ versus terminal ileum to cecum placement $(59 \%)$ at $88 \%$ cure $(95 \% \mathrm{CI}: 0.78-0.94 ; \mathrm{P}<0.001)$ and cecum to ascending colon $(28 \%)$ at $86 \%$ cure (95\% CI: $0.63-0.95$; $\mathrm{P}<0.006)$. Studies that specified the use of loperamide after FMT $(21 \%)$ had a cure of $85 \%$ (95\% CI: $0.63-0.95$; $\mathrm{P}<0.004)$.

Conclusion: FMT placed by colonoscopy has a role in the cure of recurrent or refractory CDI. Stopping antibiotics 24 hours prior to FMT results in higher percentage cure (95\%). Distribution of FMT throughout the colon has better outcomes than FMT instillation at other locations. Effect of loperamide post-FMT placement is not conclusive due to the low percentage of reported use. Prospective studies are recommended to study these factors for confirmation of effects.

\section{Six-Year Experience of Influenza Vaccination as a Condition of Employment for a Large Regional Health Care System}

John R. Brill, Mark Hermanoff, Angela Tonozzi, Mary Jo Capodice, Jennifer Farrar, Zarina Dawoodbhai

Departments of Population Health, Immunology, Infection Control, Occupational Health, Employee Health, and Practice Optimization, Aurora Health Care 